\title{
Trampas en educación física. ¿por qué se hacen y cómo podemos evitarlas?
}

Cheating in physical education. Why is this done and how can we avoid it?

\section{Pablo Sánchez Pérez ${ }^{1}$}

${ }^{1}$ Licenciado en Ciencias de la Actividad Física y del Deporte por la Universidad de León (España). Profesor de educación física en la Junta de Andalucía, España.

\section{Detalles del artículo:}

Número de palabras: 4.026; Tablas: 1; Figuras: 10; Referencias: 12

Recibido: julio 2020; Aceptado: agosto 2020; Publicado: abril 2021

Conflicto de interés: El autor declara que no existen conflictos de interés.

Correspondencia del autor: Pablo Sánchez Pérez, psancp00@gmail.com

\section{Resumen}

Introducción. ¿Por qué el alumnado hace trampas en el transcurso de las tareas y juegos en educación física? ¿Qué les lleva a ello? ¿Cómo podemos gestionar la clase para evitar su realización? Se realiza un análisis y estudio sobre cómo el alumnado piensa en lo referido a las trampas en los juegos y tareas. Para que los discentes no vean en ello como un recurso válido y útil, se ha de orientar correctamente la actuación educativa del equipo docente. Pero, además, también del entorno familiar y social de los propios adolescentes. Estas situaciones de engaños que se llevan a cabo en el ámbito escolar están relacionadas directamente con la vida cotidiana de nuestros alumnos ya que, en pleno proceso educativo, el que ellos mismos se den cuenta de que "el rendimiento por encima de las reglas" no es lo más valorable desde el punto de vista del docente para una mejora de resultados académicos, facilitará que se disipe la idea de que su práctica es beneficiosa. Objetivos. Por ello, los objetivos que se persiguen son: (I) Conocer los motivos que llevan al alumnado a hacer trampas, (II) conocer cómo actuaría el propio alumnado frente a estas situaciones y (III) determinar un enfoque correcto en educación física para evitar estas situaciones. Método. Se lleva a cabo una recogida de datos a través de dos cuestionarios, con seis preguntas ambos, en donde por un lado se intenta descubrir los motivos que llevan a los discentes a llevar a cabo este tipo de prácticas y por el otro, cómo actuarían ellos en el caso de que fueran los encargados de gestionar las trampas en el transcurso de las sesiones de educación física. Resultados. Principalmente, el alumnado destaca que los motivos por los cuáles realizan o son propensos a realizar trampas están relacionados con sus pensamientos de que los resultados obtenidos en las diferentes tareas marcan su resultado académico. Además, se inclinan por la vertiente de sancionar mediante partes u otro tipo de sanciones al alumnado que las ponga en práctica. Conclusiones. Para acabar, se concluye que la orientación de no sólo la educación en sí, sino de la educación física en particular, marca el aumento o bajada de la probabilidad de que el alumnado tome consciencia de que el llevar a cabo trampas en las actividades no es necesario para obtener un buen resultado académico y un feedback positivo por parte tanto del profesorado como de sus compañeros.

Palabras clave: Metodología, trampas, educación física, tareas, proceso educativo 


\section{Abstract}

Introduction. Why do students cheat during Physical Education tasks and games? What makes them do that? How can we deal with classroom management in order to prevent this from taking place? An analysis and study are carried out on how students think with regard to cheating in games and tasks. In order for students not to see this as a valid and useful resource, the educational performance of the teaching team, family and social environment of adolescents must be guided correctly. These cheating situations carried out in the school environment are directly linked to the daily life of our students as in the midst of the educational process, the fact that they realize that the reasons that can lead to cheating are not valued on the part of the teacher for an improvement in academic performance, will aid to dispel the idea of practising it. Objetives.Therefore, the objectives pursued are threefold: (I) To discover the reasons that lead students to cheat (II) discover how students would act in these situations and (III) determine an appropriate approach in physical education to avoid these situations. Method. Data is collected by means of two questionnaires, each containing six questions, which seek to discover the reasons for students cheating and how they would act in the case of being responsible for managing cheating during physical education sessions. Results. Mainly, students highlight that the reasons for carrying out or for being prone to implement cheating are related to thinking that their academic performance springs from the different marks they obtain in each task. Moreover, they are inclined to sanction students who cheat, and the types of sanctions can vary. Conclusions. Finally, it is concluded that the focus of not only education itself, but specifically Physical Education, marks the increase or decrease of the probability that students become aware that cheating is not necessary to obtain a good academic performance and positive feedback from both teachers and students' peers.

Key words: Methodology, cheats, physical education, tasks, educational process

\section{INTRODUCCION}

Uno de los pilares de la educación en general debe ser el respeto a personas, así como a las normas. No hay que olvidar que en el proceso educativo estamos formando personas para convivir en sociedad. Ésta se caracteriza por continuas interacciones, dentro de las cuáles se han de respetar unos principios, normas y valores, de manera que sean positivas evitando que sucedan momentos de tensión y desacuerdo entre los componentes de dicha interacción (Sáez de Ocáriz, Lavega y March, 2013).

Concretamente en Educación Física, hemos de ser conscientes de la necesidad de interiorizar y automatizar en el alumnado un comportamiento acorde a unos niveles de respeto, solidaridad, cooperación y cumplimiento de sus reglas (Gil et al., 2007). Esto va a concurrir en una mejora del ambiente del grupo clase y de un mejor aprovechamiento del tiempo efectivo de cada sesión impartida.

Por ello, y dado que día a día se producen conflictos en el transcurso de las diversas tareas por momentos en que ciertos alumnos intentan hacer trampas para obtener ventaja de las mismas, se lleva a cabo este estudio que pueda ayudar a los docentes a prevenirlas una vez que se conocen los principales motivos que llevan a los discentes a ello. 
Sánchez Pérez, P. (2021). Trampas en educación física. ¿Por qué se hacen y cómo podemos

evitarlas?. Journal of Physical Education and Human Movement,

$3(1), 10-35$

Para poder incidir en los elementos a tener en cuenta y en donde el profesorado o escalas más altas deben de prestar atención, es necesario que se analicen los mismos. ¿Cómo lo analizo? Ayudándome de las opiniones de los alumnos (Gil et al., 2007). Éstos son capaces de realizar un análisis acerca de lo que perciben en la ejecución de tareas y juegos, además de ser conscientes de las necesidades para gestionar las trampas y salto de las normas en los mismos.

\section{MATERIAL Y MÉTODOS}

Para llevar a cabo este estudio se tomó una muestra de 130 alumnos de la etapa de Educación Secundaria Obligatoria, dentro del intervalo de 12 a 16 años de edad, pertenecientes al I.E.S Santa María La Real de la localidad de Aguilar de Campoo, Palencia.

En la siguiente tabla se descompone la muestra por cursos:

\begin{tabular}{|c|c|c|c|c|}
\hline ALUMNOS & $\mathbf{1}^{\mathbf{0}}$ E.S.O & $\mathbf{2}^{\mathbf{0}}$ E.S.O & $\mathbf{3}^{\mathbf{0}} \mathbf{E . S . O}$ & $\mathbf{4}^{\mathbf{0}} \mathbf{\text { E.S.O }}$ \\
\hline Total & 45 & 41 & 12 & 32 \\
\hline Chicas & 23 & 22 & 8 & 16 \\
\hline Chicos & 22 & 19 & 4 & 17 \\
\hline
\end{tabular}

Tabla 1. Descomposición de participantes encuestados por curso académico.

Estos alumnos rellenaron dos cuestionarios de manera anónima. El cuestionario 1.1 "PQHT" hace referencia a la percepción del alumnado y la justificación del ¿por qué haces trampas?, mientras que el cuestionario 1.2 "OVEP" se orienta hacia el comportamiento del profesorado frente a las situaciones de trampas en los juegos y tareas en Educación Física. Así, este segundo cuestionario nos indica la percepción del alumnado frente a situaciones que se dan y sobre si la norma debe hacerse respetar.

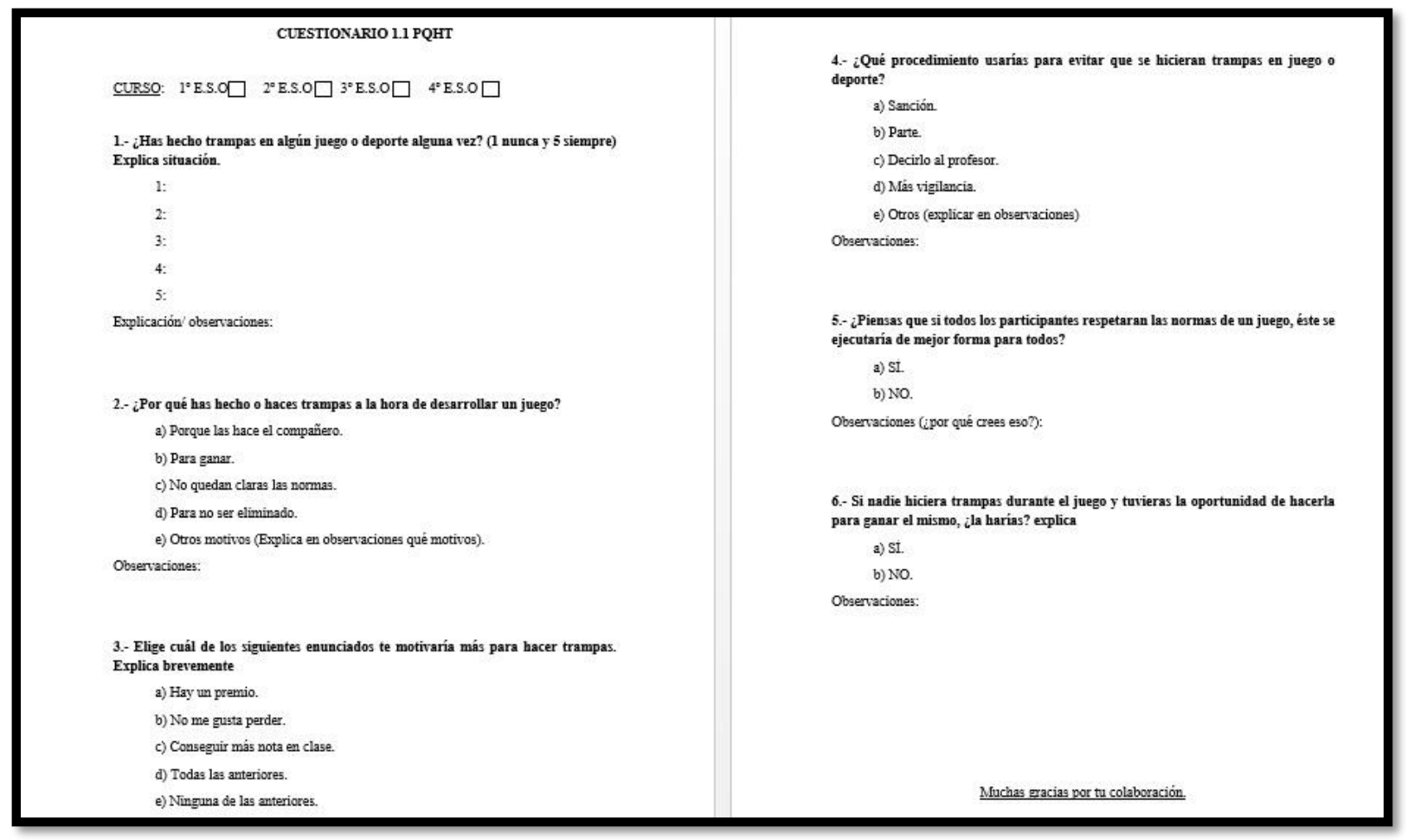

Figura 1: Cuestionario 1.1 “PQHT” (“¿Por qué haces trampas?”). 


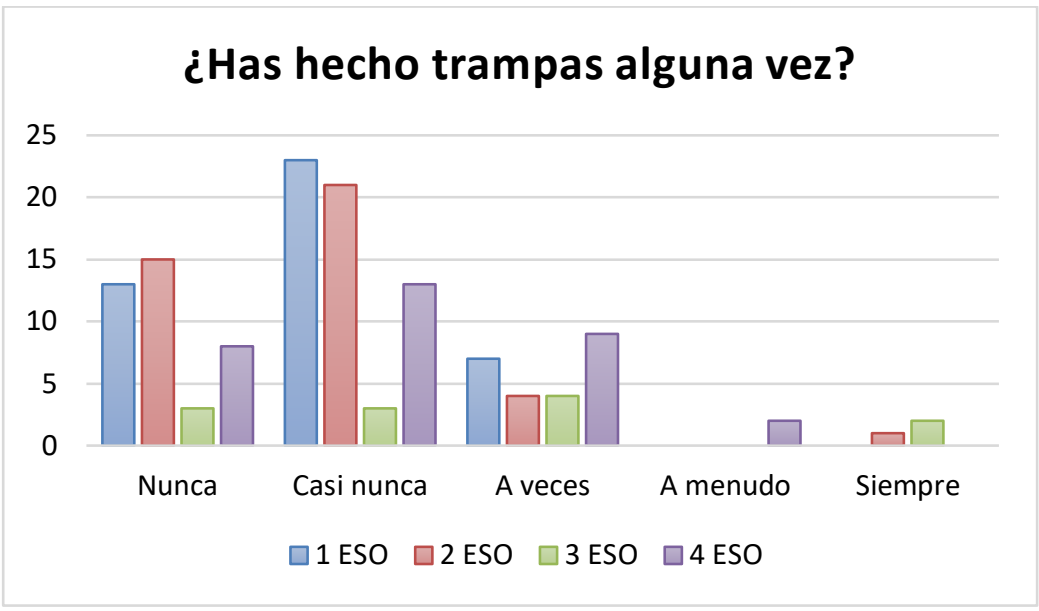

Figura 3. Resultados pregunta 1 del cuestionario 1.1 "PQHT".

Cabe destacar que en dicha pregunta, únicamente 5 alumnos/as (pertenecientes a $3^{\circ}$ y $4^{\circ}$ E.S.O), afirman haber hecho trampas muy a menudo o siempre que es posible. Este grupo supone únicamente un $3.9 \%$ del total, por lo que hace ver que a priori hay una intención positiva de cara a no practicar trampas en las tareas puestas en prácticas. Como bien se intenta sensibilizar al alumnado desde la aplicación de la antigua LOGSE (1990) hay que priorizar el trabajo sobre la conciencia de la educación en valores (Gil-et al., 2007), dando lugar a que en un futuro este tanto por ciento $(\%)$ de respeto a la norma y evitar la aparición de trampas sea mayor y más extendido entre los adolescentes.

Respecto a la pregunta “¿qué enunciado te motiva para hacer trampas?”, el 31,9\% elige "para subir nota" y el 21,9\% "hay un premio", destacando el carácter cuantitativo de lo que supone la calificación en el sistema educativo actual o la necesidad por parte del discente de ser recompensado por sus resultados o rendimiento. No obstante, tal como reseñan Alonso, Etxebeste y Lavega (2010), las emociones positivas se ven elevadas cuando aparece la competición. Por ello, los docentes nos vemos en la obligación de enseñar al alumnado el ideal de competición sana y el aceptar el resultado sin que ello suponga intentar saltarse las reglas para llegar a la victoria.

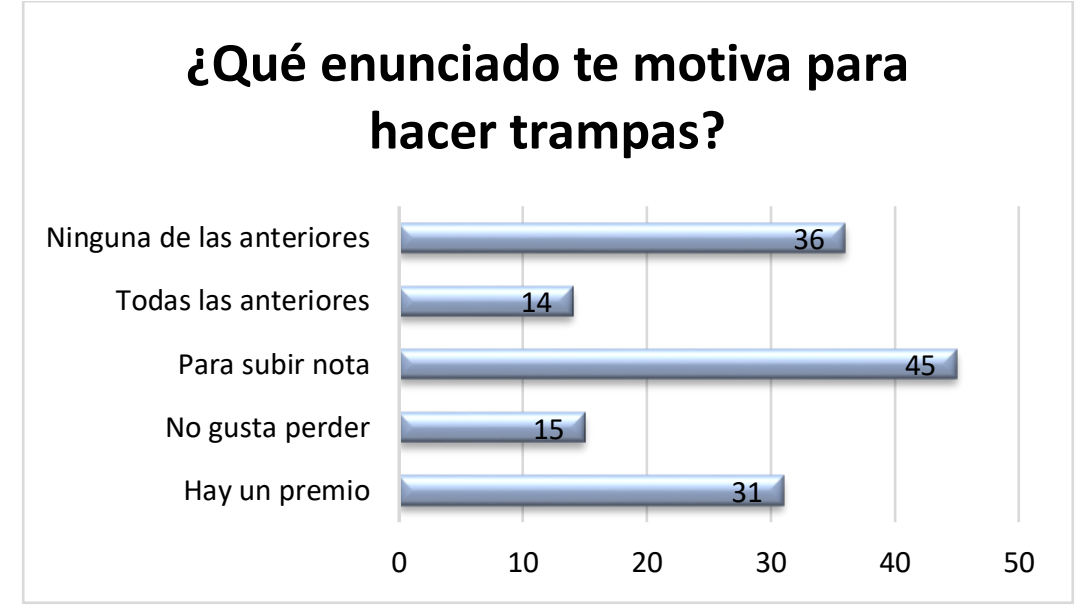

Figura 4. Resultados pregunta 4 del cuestionario 1.1 "PQHT". 
Por otro lado, un $25.5 \%$ de ellos, responde que ninguna respuesta le motivaría ya que no se pensarían el hacer trampas. Esto se relaciona con la pregunta anterior en donde únicamente 3 alumnos más expusieron que nunca habían hecho trampas. Cabe destacar, que el hecho de "hacer trampa" no se soluciona ni puede ser reconducido en una única situación, sino que es un proceso progresivo en donde el objetivo es que el alumnado tome consciencia y sea capaz de por sí mismo de desechar este hábito (Agüero- San Juan, 2015).

Sobre los motivos del por qué se hacen trampas, con gran diferencia y destacada, está la respuesta de "para ganar". En ella se apoyan 48 de los 80 alumnos/as que han contestado a esta pregunta $(60 \%)$.

Con un porcentaje más reducido estarían las respuestas referidas a: "para no ser eliminado", "sin querer" y "de broma". Quizás una forma en la cual el alumnado no vea la necesidad de ganar como lo primordial y prioritario es trabajar los distintos contenidos mediante trabajos cooperativos y trabajos en equipo en donde el objetivo sea grupal y donde la cooperación, solidaridad y esfuerzo sean aquellos parámetros que primen (González y Del Valle, 2006).

A pesar de que ello pueda suponer un inicio en la educación en valores dentro de los contenidos de la Educación Física, poco a poco se debe de ir introduciendo la competición sana como recurso educativo en nuestras aulas para llegar a esa formación integral que se persigue mediante la legislación educativa vigente (LOMCE, 2013).

\section{MOTIVOS DEL POR QUÉ SE HACE TRAMPAS}

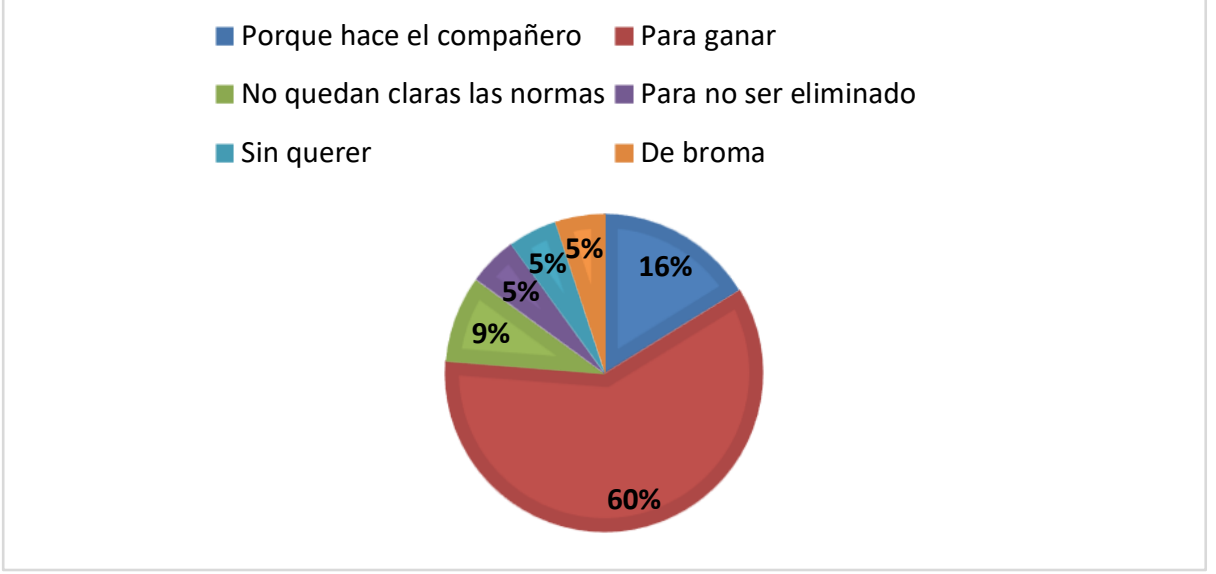

Figura 5. Resultados pregunta 2 del cuestionario 1.1 "PQHT".

También se le pregunta por el procedimiento que ellos piensan que se debería llevar a cabo para evitar que se hagan trampas en el transcurso de los diferentes juegos o tareas. De los 118 alumnos/as que responden a esta pregunta, el 38,6\% expone que una sanción sería lo ideal (no se concreta qué tipo de sanción). Otras dos respuestas que han tenido amplia repercusión con 22 y 29 alumnos son respectivamente imponer un castigo o parte (que pueden sumarse a los que otros discentes llaman "sanción"), y que exista más vigilancia tanto por parte del profesor como por parte del alumnado, tomando roles de árbitros o jueces. 
Esto último se ampara o se ve motivado en una de las metodologías competenciales actuales como es la metodología de educación deportiva. Aunque ésta puede ser una de las maneras preventivas para evitar las trampas, no sería una opción infalible, ya que los supuestos en los que se da la trampa, el engaño tiene como finalidad incorporada la de engañar a esta figura que actúa como juez, árbitro o controlador para que sea efectiva (Agüero- San Juan, 2015).

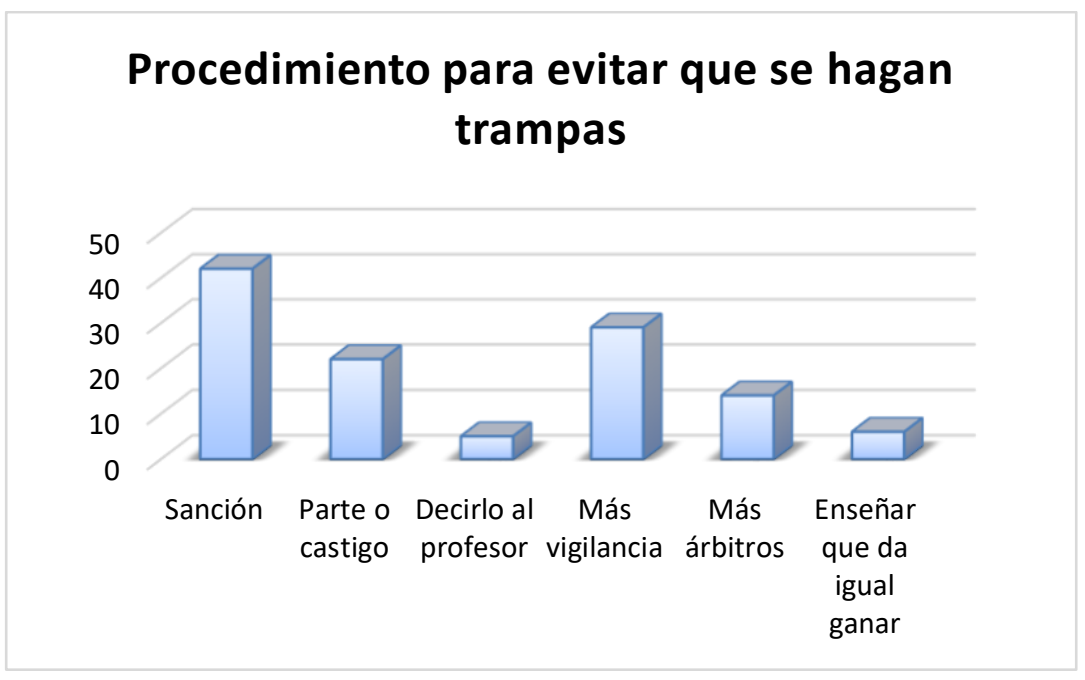

Figura 6. Resultados pregunta 5 del cuestionario 1.1 "PQHT".

En la quinta pregunta, en la que se cuestiona si sería mejor o no que todos los participantes respetasen las normas, 129 alumnos responden a ella con clara tendencia a que sí sería mejor, con un $94.6 \%$ de respuestas. Ello refleja que las actividades deportivas, bien utilizadas y bien orientadas, promueven el respeto a las normas (Gil et al., 2007). Ya que la persona no se ve en la obligación inicial de llevarlas a cabo, sino que el transcurso del juego o tarea orientada de una forma resultadista, es lo que lleva a incentivar este tipo de prácticas.

Para terminar con este primer cuestionario, valorando la ética de realizar o no trampas si el resto no las hace, de 129 respuestas, el 76,7\% de ellas reconocen que sí harían trampas para ganar.

\section{SI NADIE HACE TRAMPAS, ¿LAS HARÍAS PARA GANAR?}

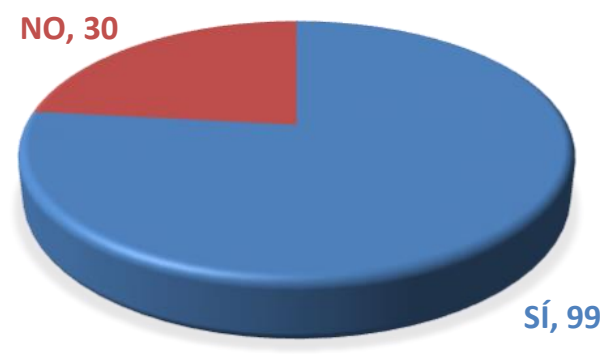

Figura 7. Resultados pregunta 6 del cuestionario 1.1 "PQHT". 
Esta pregunta se relaciona con la tercera de este cuestionario, donde el ganar o mejor dicho, el ganar por encima de todo, parece que a los adolescentes les hace pensar que de esta forma se les valorará mejor o tendrá una mejor consideración por parte de su entorno más próximo.

2) Si el alumnado fuera el profesor/a, ¿Cómo actuaría frente a estas situaciones?

En relación con el test 1.2 "OVEP" (“otra visión es posible"), al alumnado se les plantea diferentes cuestiones cobre cómo actuarían siendo ellos profesores de Educación Física o cómo creen que un profesor de dicha materia debería de actuar.

En primer lugar, en la pregunta explícita de que haría si fuera profesor de Educación Física, de 130 alumnos que contestan, el 90\% expone que sancionaría el no cumplimiento de las normas. Esto va correlacionado con el sentimiento de respeto a los demás y sobre sí mismo que la persona tiene sobre si alguien es capaz de saltarse las normas y así, poder modificar el transcurso de la tarea a su favor. El alumnado se va formando, como hemos dicho, para vivir en sociedad y ello exige el respeto a las normas que se dan dentro de la interacción con los demás en distintos ámbitos sociales y de la vida (Salas, 2010). Sólo 10 de ellos optaría por eliminar las reglas y únicamente 3 contestarían que el resultado importaría más que el proceso educativo.

\title{
EN RELACIÓN A LAS NORMAS, SI FUERAS PROFESOR DE EF, ¿QUÉ HARÍAS?
}

\author{
Sancionar no cumplimiento \\ Eliminar reglas \\ - Importaría más el resultado que proceso
}

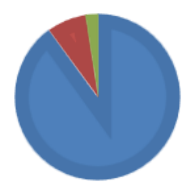

Figura 8. Resultados pregunta 1 del cuestionario 1.2 "OVEP”.

De ello se entiende que la gran mayoría entendería y apoyaría a que se realizaran acciones en contra de la realización de trampas durante las tareas.

En lo referido a la percepción que tienen sobre qué ocurre en sus clases de Educación Física, sobre si el profesorado sanciona o no estas situaciones de trampas que se pueden dar, el $78,5 \%$ de 130 alumnos que responden afirman que sí se sancionan. 12 de ellos (9\%) dice que el profesorado no interviene y un $12 \%$ refleja que según quien sea el alumno/a que se salte las normas, el profesor actúa o no. 


\section{RESPECTO A LAS NORMAS DE LAS DISTINTAS TAREAS EN EF...}

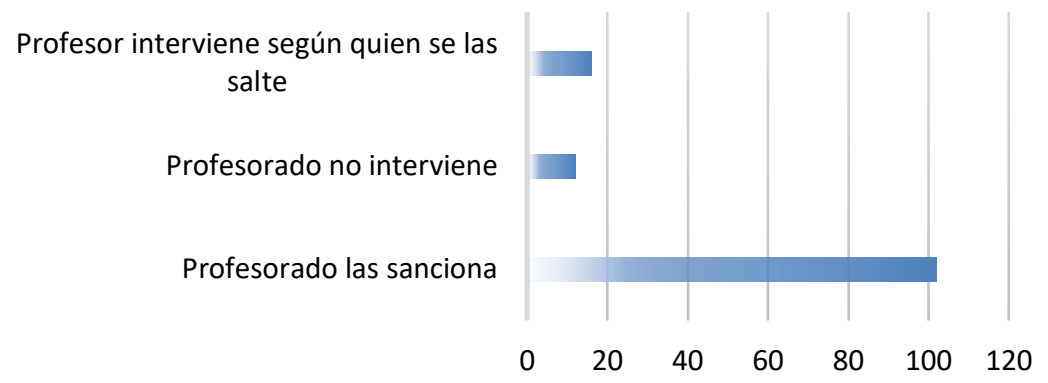

Figura 9. Resultados pregunta 4 del cuestionario 1.2 "OVEP".

La mayoría del alumnado que responde a la siguiente pregunta (84 alumnos, que suponen $64.6 \%$ del total), afirma que cuando un compañero se salta las normas y por ello el resultado de la tarea o juego cambia, se quejan al profesor/a. 31 de ellos reflejan que no les importa y 15 de ellos intentan hacer trampas como contra a la acción del compañero.

Dentro de la pregunta "En EF, ¿por qué crees que es importante el seguir las normas?", un $30 \%$ se inclinan por la opción de que ayuda al buen funcionamiento de la tarea y un $17 \%$ piensan que de esa manera todo es más justo.

En una cantidad algo más pequeña se reparten el resto de respuestas. Un 17,7\% que contestan la opción "otros", reflejan aspectos como el respeto, eficiencia del proceso de aprendizaje, etc.

En definitiva, el respeto a la norma conlleva una buena dinámica de la tarea, del grupo clase (Salas, 2010) y por ende, de un tratamiento de elementos transversales como es la educación para la paz (LOMCE, 2013).

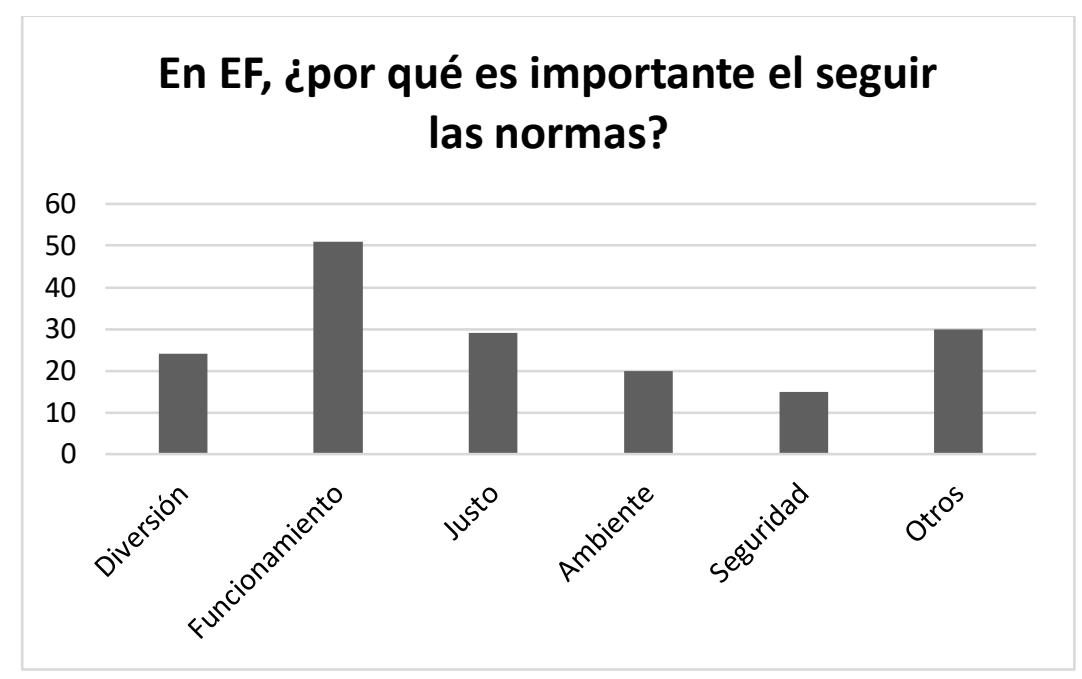

Figura 10. Resultados pregunta 5 del cuestionario 1.2 “OVEP”. 
Esta muestra de alumnos/as se inclina a que la mayoría de los discentes respetan las normas de las diferentes tareas en Educación Física, con un 73\% del total de respuestas, lo que se refiere a 95 alumnos. 24 discentes piensan que la mayoría se las saltan $(18,5 \%)$ y únicamente 11 alumnos/as $(8,5 \%)$ cree que el alumnado no tiene las normas en cuenta a la hora de llevar a la práctica la actividad.

Para acabar, incentivando de nuevo el carácter moral de qué creen sobre el respeto a las normas de los juegos, 121 de 130 alumnos que contestan a esta pregunta (93\%) piensan que todos deberían respetarlas; y únicamente 5 y 4 alumnos creen que no son importantes seguirlas o que alguno de cada equipo podría saltárselas, respectivamente.

3) ¿Cuál debería ser el enfoque correcto en educación física para evitar estas situaciones?

Uno de los principales objetivos que debe perseguir esta materia es que además de potenciar e incorporar la actividad física y hábitos saludables en el alumnado es el de formar personas que sepan vivir y convivir en sociedad. De hecho, numerosos autores destacan y valoran la materia de Educación Física como la idónea para contribuir al desarrollo formativo positivo del alumnado (García, 2017).

Para contribuir a ello, aparecen nuevas metodologías, como por ejemplo el modelo de responsabilidad personal y social (Sánchez Alcaraz, Gómez Mármol, Valenzuela, Sánchez y Díaz, 2016), así como el modelo de educación deportiva (Méndez Giménez, Fernández- Río y Méndez- Alonso, 2015).

Con estos tipos de enfoque lo que se pretende es que los discentes aprendan, adquieran o mejoren valores útiles para su desarrollo como persona. Entre ellos destaco, el respeto por las normas, por los compañeros, adversarios y aprender que no todo es válido para conseguir el propósito.

No obstante, además de éstas y más metodologías que actualmente se llevan a la práctica, la forma en la que el docente plantee las actividades, el proceso en sí y la evaluación, conllevará que sus alumnos perciban que lo que se valora no es el ganar o ser mejor que el otro, sino el propio proceso de cada uno en la adquisición de autonomía y competencias logradas a través de lo motriz (Blázquez, 2010).

Autores como Contreras (2009), destacan que la Educación Física es, ante todo, educación, resaltando en que su particularidad es que se opera mediante el movimiento. Es decir, no es educación de lo físico, sino educación de la persona por medio de la motricidad. Esto, es lo que debemos proponernos conseguir en el proceso educativo de cada alumno/a.

\section{CONCLUSIONES}

Cabe destacar la claridad en los datos de los cuestionarios que arrojan cierta luz sobre el porqué se suelen llevar a cabo ciertas prácticas no adecuadas en las tareas y cómo modificando la percepción del objetivo educativo se puede conseguir una sociedad más justa, solidaria y respetuosa con las reglas.

Las trampas en no sólo el contexto de la Educación Física, sino en su extrapolación a la vida cotidiana, supone un problema para el avance a una mejor sociedad. La mayoría del 
alumnado reconoce que alguna vez ha hecho alguna trampa, de mayor o menor calibre; aunque lo más llamativo es el motivo competitivo o reforzador que les lleva a cometerlas.

El subir nota, el haber un premio o simplemente el poder ganar, no hace más que reforzar la idea de llevar a la práctica una Educación Física cuya principal motivación a practicarla sea meramente intrínseca, de tal manera que el alumnado inmerso en su propio proceso de enseñanza aprendizaje, no se vea en la necesidad de realizar ningún tipo de trampas o engaño con el fin de mejorar sus resultados académicos. También es preciso resaltar que el propio alumnado ve necesaria la sanción, parte o castigo sobre situaciones donde alguien realiza este tipo de actos.

Hay una relación estrecha entre el correcto enfoque de nuestro proceso educativo y el que el alumno vea innecesario la realización de trampas. Por ello, se insiste en que la idea de todos los estamentos de la sociedad inmersos en la educación debe ser capaces de iniciar y/o avanzar en el camino de convertir una educación tradicional competitiva en una más formativa e individualizada a efectos de los objetivos perseguidos y que conecte con el actual enfoque del sistema y ley educativa, llevar a cabo un aprendizaje significativo y competencial.

Como enfoque metodológico correcto para llegar a este tipo de aprendizaje, se resaltan la educación deportiva y el modelo de responsabilidad personal y social. No obstante, el abanico de metodologías y recursos educativos es tan amplio que son meros ejemplos de todo aquello que el docente puede intentar llevar a cabo para cumplir con los objetivos a alcanzar. 


\section{REFERENCIAS}

Alonso, J. I., Etxebeste, J. y Lavega, P. (5- 8 de octubre de 2010). Análisis de emociones suscitadas en juegos motores con y sin competición. VI Congreso Internacional de la Asociación Española de Ciencias del Deporte, Universidad Miguel Hernández, Elche, España.

Blázquez, D. (2010). Evaluar en educación física. Inde, Barcelona.

Contreras, O. (2009). Didáctica de la educación física. Un enfoque constructivista. Inde, Barcelona.

García Fernández, J. M. (2017). La Educación en valores en educación física. Análisis de los comportamientos en los alumnos de Educación Primaria. Revista digital docente: Campus Educación, 6, 13-17

Gil Madrona, P., Lorente, P., Paterna Martínez, A., Padilla, M., Martínez, G., Sánchez, J., Zamorano-García, D., García, G., Chicano, A. y Pastor Vicedo, J. (2007). ¿Cómo educar en valores en Educación Física a través de juegos y deportes?

González, P.J. y Del Valle, M.C. (2006). La educación en valores desde el área de educación física: una propuesta práctica para Educación Primaria. Revista Digital educación física y deportes, 101

Ley Orgánica 8/2013, de 9 de diciembre, para la Mejora de la Calidad Educativa

Méndez- Giménez, A., Fernández- Río, J. y Méndez- Alonso, D. (2015). Modelo de educación deportiva versus modelo tradicional: Efectos en la motivación y deportividad. Revista Internacional de Medicina y Ciencias de la Actividad Física y del Deporte, 15 (59), 449- 466.

Sáez de Ocáriz, U., Lavega, P. y March, J. (2013). El profesorado ante los conflictos en la educación física. El caso de los juegos de oposición en Primaria. Revista Electrónica Interuniversitaria de Formación del Profesorado, 16 (1), 163- 176. http://dx.doi.org/10.6018/reifop.16.1.180101

Salas Fernández, M. C. (2010). Valores de la educación física dentro y fuera de la escuela. Revista Digital educación física y deportes, 150

Sánchez-Alcaraz, B., Gómez-Mármol, A., Valenzuela, A., Sánchez, E. y Díaz Suarez, A. (2016). El Modelo de Responsabilidad Personal y Social a través del deporte como propuesta metodológica para la educación en valores en adolescentes. Espiral. Cuadernos del Profesorado. 9. 16-26. 10.25115/ecp.v9i18.997.

Sebastián Agüero-San Juan (2015): El concepto de hacer trampa en el deporte organizado. Un inventario temático de problemas. Fair Play. Revista de Filosofía, Ética y Derecho del Deporte, 3:2, 154-181 